\title{
Effect of the Fructus Ligustri Lucidi extract and its monomers quercetin and oleanolic acid on the adhesion and migration of melanocytes and intracellular actin
}

\author{
YANHUA WU, QILIN LI, XIANGJUN LI, DANHUA HE, MU NIU, XIAOJUAN LU and HUI LI \\ Department of Dermatology, Guangzhou Red Cross Hospital, Medical College, \\ Jinan University, Guangzhou, Guangdong 510220, P.R. China
}

Received December 14, 2015; Accepted February 23, 2016

DOI: $10.3892 /$ br.2016.638

\begin{abstract}
The present study aimed to investigate the effects of the Fructus Ligustri Lucidi (FLL) extract and its monomers quercetin and oleanolic acid on the adhesion and migration of human epidermal melanocytes (MCs) and intracellular actin. The human epidermal MCs were cultured and identified. The cells were treated with different concentrations of FLL extract, quercetin and oleanolic acid. The adhesion and migration abilities of the cells were determined by the fibronectin-coated culture experiment and Transwell assay, respectively. The structure and distribution of intracellular actin were observed by confocal laser microscopy, with semi-quantitative analysis. Results showed that compared with the control group, $0.0375-0.3 \mathrm{mg} / \mathrm{ml}$ of the FLL extract and $40 \mu \mathrm{M}$ quercetin significantly improved the adhesion rate of MCs $(\mathrm{P}<0.05)$. The numbers of MCs permeating the microporous membrane in the $0.15 \mathrm{mg} / \mathrm{ml} \mathrm{FLL} \mathrm{extract} \mathrm{and} 12 \mu \mathrm{M}$ oleanolic acid groups were 43.7 and 30.3, respectively, significantly higher compared to the control group $(\mathrm{P}<0.01)$. In the control group, the intracellular actin was less, and the stress fiber structure was not clear. In the $0.15 \mathrm{mg} / \mathrm{ml}$ FLL extract, $12 \mu \mathrm{M}$ oleanolic acid and $40 \mu \mathrm{M}$ quercetin groups, there were numerous bunched stress fibers, indicating the aggregation of filamentous fibrous actin. The mean optical densities of actin expression in the $0.15 \mathrm{mg} / \mathrm{ml}$ FLL extract, $12 \mu \mathrm{M}$ oleanolic acid and $40 \mu \mathrm{M}$ quercetin groups were significantly higher compared to the control group $(\mathrm{P}<0.05)$. The FLL extract has a significant stimulatory effect on the adhesion and migration of human epidermal MCs. The mechanism may be associated with the promotion of intracellular actin cytoskeleton aggregation.
\end{abstract}

Correspondence to: Dr Qilin Li, Department of Dermatology, Guangzhou Red Cross Hospital, Medical College, Jinan University, 39 Tongfu Zhong Road, Guangzhou, Guangdong 510220, P.R. China E-mail: qilinlicn1@126.com

Key words: Fructus Ligustri Lucidi, extract, melanocytes, adhesion, migration, actin

\section{Introduction}

A previous study has shown that the disequilibrium of the microenvironment in melanocytes (MCs) and the regression of cell adhesion and migration, which are caused by psychological, nerve and immunity factors, are the main pathogeneses of vitiligo (1). In the initial effective stage of vitiligo treatment, the pigmentation occurs from the hickie follicular orifice, and extends to be flakiness. There are amelanotic melanocytes (AMMCs) in the hair follicle outer root sheath, which are the most important $\mathrm{MC}$ reservoir for skin lesion repigmentation (2). During the repigmentation course, MCs experience the stage of activation, migration, adhesion and proliferation (3). The dynamic equilibrium of aggregation and disaggregation of actin cytoskeleton is the important regulating factor for cell adhesion and migration.

Fructus Ligustri Lucidi (FLL), as a member of Chinese herbs, has an important role in the treatment of disease. The active ingredients of FLL include oleanolic acid, butyl alcohol, tyrosol, quercetin, palm acid, stearic acid, polysaccharide, oleic acid, flax and linum (4). Quercetin and oleanolic acid are two most important monomers of FLL extract. Oleanolic acid has the anti-inflammatory, antimutagenic, antioxidant, anti-virus and liver protection activity $(5,6)$. Quercetin has the function of antioxidation, anti-inflammation, lowering blood pressure, anti-platelet aggregation, antitumor, anti-atherosclerosis and regulating immunity (7-9). Previous studies have reported that the FLL extract and its monomers oleanolic acid and quercetin can improve the tyrosinase activity of MCs, and stimulate the melanogenesis (10-14). However, their effects on adhesion, migration of MCs and the intracellular actin are seldom reported. In the present study, the effects of the FLL extract and its monomers oleanolic acid and quercetin on the adhesion and migration of human epidermal MCs and intracellular actin were investigated, and the associated mechanism was discussed. The aim was to provide a basis for further application of FLL to treatment of vitiligo.

\section{Materials and methods}

Materials. The FLL extract was prepared by ethanol extraction from FLL (China's National Institute for the Control of 
Pharmaceutical and Biological Products, Beijing, China). FLL was soaked in $10 \mathrm{ml}$ of $90 \%$ ethanol (Sigma-Aldrich, St. Louis, MO, USA) for 1 week at room temperature. Following filtration, the extraction solution was obtained. The ethanol was removed by concentration, and the cream mixture was obtained. The $200 \mathrm{mg} / \mathrm{ml}$ ethanol solution of the FLL extract was prepared. Using fresh M254 medium (Fuzhou Maixin Biotechnology Development Co., Ltd., Fuzhou, China), the concentration of the FLL extract was adjusted to 0.6, 0.3, 0.15, 0.075 and $0.0375 \mathrm{mg} / \mathrm{ml}$, respectively. Quercetin and oleanolic acid were provided by China's National Institute for the Control of Pharmaceutical and Biological Products. The concentration of quercetin was adjusted to $80,40,20,10$ and $5 \mu \mathrm{M}$, respectively, and the concentration of oleanolic acid was adjusted to $24,12,6,3$ and $1.5 \mu \mathrm{M}$, respectively.

Culture and identification of MCs. The circumcision specimens were obtained from 10-25-year-old normal adolescent males who underwent circumcision in the Department of Urology (The Fourth Affiliated Hospital of Jinan University, Guangzhou Red Cross Hospital, Guangzhou, Guangdong, China). The patients had no history of pigmentation disorder such as vitiligo. The study was approved by the ethics committee of the Fourth Affiliated Hospital of Jinan University. Written informed consent was obtained from the patients. The circumcision specimens were soaked in $70 \%$ alcohol for 5-10 min, followed by rinsing with phosphate-buffered saline (PBS; Sigma-Aldrich) 6-12 times. The specimens were transferred to the sterile petri dish containing a small amount of PBS. The dermis and subcutaneous fatty tissue were removed. The remaining specimens were separated into square pieces $(2 \times 2 \mathrm{~mm})$, and tiled in the sterile petri dish. $0.25 \%$ Dispase II (Fuzhou Maixin Biotechnology Development Co., Ltd.) was added for digestion at $4^{\circ} \mathrm{C}$ for $16-24 \mathrm{~h}$, followed by incubation at $37^{\circ} \mathrm{C}$ for $1 \mathrm{~h}$. The epidermis was separated and was washed with PBS 3-5 times. Following digestion for 5 min, M254 complete medium containing 10\% fetal calf serum (Fuzhou Maixin Biotechnology Development Co., Ltd.) was added to terminate digestion. The MCs were pipetted from the epidermis, and the cell suspension was obtained, followed by filtering with a 200-mesh sieve (Fuzhou Maixin Biotechnology Development Co., Ltd.). Following centrifugation at $225 \mathrm{x}$ g for $3 \mathrm{~min}$, cell counting was performed. After $24 \mathrm{~h}$ of culture using M254 culture medium (Fuzhou Maixin Biotechnology Development Co., Ltd.), the first culture medium was changed, followed by a change of culture medium every 3 days. After primary culture for 8-15 days, the MCs were $80 \%$ confluent. The cells were inoculated to the new sterile flasks for continued culture. The 3-5 generation of MCs were used for further experiments.

MCs were identified by the L-DOPA staining and HMB-45 immunochemical staining method. For the former method, the cell climbing slice was prepared and was fixed with 4\% paraformaldehyde (Sigma-Aldrich) for $25 \mathrm{~min}$. The slice was incubated in L-DOPA staining solution (Shanghai Sangon Biological Engineering Co., Ltd., Shanghai, China) for $4 \mathrm{~h}$, followed by washing with water for $5 \mathrm{~min}$. For the latter method, the cell climbing slice was fixed with $4 \%$ paraformaldehyde for $25 \mathrm{~min}$, followed by washing with PBS for 5 min. The mouse anti-human HMB-45 monoclonal antibody (cat no. SG7870; used 1:50; Shanghai Sangon Biological
Engineering Co., Ltd.) was used as the primary antibody, and the anti-mouse IgG-HRP (cat no. SC2023; used 1:500; Santa Cruz Biotechnology, Inc., Santa Cruz, CA, USA) was used as the secondary antibody. The diaminobenzidine coloration, counterstain, dehydration, transparency and mounting were performed according to the manufacturer's protocol. PBS replacing the primary antibody was used as the negative control.

Determination of cell proliferation activity with the XTT assay. MCs were inoculated in 96 -well plates $\left(5 \times 10^{4} \mathrm{cell} / \mathrm{ml}\right)$, $100 \mu \mathrm{l}$ for each well, followed by culture for $24 \mathrm{~h}$. The complete culture medium containing different concentrations of the drugs was added. The culture medium without the drug was used as the control. After culture for 24,48 and $72 \mathrm{~h}$, the cell morphology was observed under a DVM6 optical microscope (Leica Science Lab, Berlin, Germany). XTT/PMS solution (50 $\mu \mathrm{l}$; Sigma-Aldrich) was added to the well, followed by incubation at $37^{\circ} \mathrm{C}$ for $4 \mathrm{~h}$. The absorbance value was determined at $490 \mathrm{~nm}$. The concentration without any cytotoxicity was selected for the cell adhesion and migration determination assays.

Determination of cell adhesion. MCs were cultured with complete culture medium containing different concentrations of the drugs for 3 days. M254 medium (50 $\mu \mathrm{l})$ containing $20 \mu \mathrm{g} / \mathrm{ml}$ fibronectin (Shanghai Sangon Biological Engineering Co., Ltd.) was added to each well, followed by incubation at $37^{\circ} \mathrm{C}$ for $24 \mathrm{~h}$. Following washing with PBS, the cells were inoculated in 96-well plate coated with fibronectin $\left(5 \times 10^{5} \mathrm{cell} / \mathrm{ml}\right)$, followed by incubation at $37^{\circ} \mathrm{C}$ for $4 \mathrm{~h}$. MCs without drug treatment were used as the control. The cells without adhesion were rinsed with PBS, and subsequently $50 \mu 1 \mathrm{XTT} / \mathrm{PMS}$ solution was added to the well, followed by incubation at $37^{\circ} \mathrm{C}$ for $4 \mathrm{~h}$. The absorbance value (A) was measured at $490 \mathrm{~nm}$. The cell adhesion rate was expressed as (A490 $0_{\text {treatment group }} / \mathrm{A}^{4} 40_{\text {control group }}$ ) 100.

Determination of cell migration. The Transwell microporous membrane was coated with fibronectin $(20 \mu \mathrm{g} / \mathrm{ml})$, followed by drying in the super-clean bench overnight and washing with PBS. The MCs [100 $\mu 1$ of $2 \times 10^{5}$ cell $/ \mathrm{ml}$, diluted with M254 medium containing $0.1 \%$ bovine serum albumin (BSA)] were added to the upper chamber and $500 \mu 1$ complete medium containing different drugs was added to the lower chamber. The complete medium without drugs was used as the control. After culture for $24 \mathrm{~h}$, the chamber was removed and the cells in the upper chamber surface were grazed by a swab. The cells in the lower chamber were fixed with $4 \%$ paraformaldehyde (Sigma-Aldrich) for $30 \mathrm{~min}$. The membrane was cut using an operating knife blade, followed by hematoxylin and eosin staining for $10 \mathrm{~min}$. Three visual fields were randomly selected under a microscope, and the MCs migrating to the lower membrane were counted.

Observation of structure and distribution of actin in MCs. MCs were cultured with complete culture medium containing drugs for 3 days. MCs without drug treatment were used as the control. The density of the cells was adjusted to $2 \times 10^{4}$ cell $/ \mathrm{ml}$. The cell climbing slices were prepared, followed by fixation 
with paraformaldehyde. Following rupture of the membrane at room temperature using 0.1\% Triton X-100/PBS (Fuzhou Maixin Biotechnology Development Co., Ltd.), the cells were blocked using PBS containing 1\% BSA for $30 \mathrm{~min}$. Fluorescein isothiocyanate-phalloidin ( $5 \mu \mathrm{g} / \mathrm{ml}$; Fuzhou Maixin Biotechnology Development Co., Ltd.) was added, followed by staining at room temperature for $30 \mathrm{~min}$. Following fluorescence mounting, the slices were observed with an FV1200 confocal laser scanning microscopy (Olympus Corp., Tokyo, Japan) at $488 \mathrm{~nm}$. Three visual fields were randomly selected for semiquantitative analysis fluorescence intensity. The mean optical density (MOD) was expressed as total cell integral optical density/total cell area.

Statistical analysis. All the statistical analyses were carried out using SPSS 17.0 software (SPSS Inc., Chicago, IL, USA). Data are presented as mean \pm standard deviation. Comparisons between two groups were performed using Student's t-test. $\mathrm{P}<0.05$ was considered to indicate a statistically significant difference.

\section{Results}

Culture and identification of normal MCs. Following culture, the adherent MCs presented slender dendrites, with fusiform shape or polygon shape. The proportion of caryon to endochylema was large. There was strong halation around the cell body caused by refraction, and the cells were reticulated. Following L-DOPA staining, the endochylema and dendrite presented brown or black (positive staining) (Fig. 1A). The HMB-45 immunocytochemical stain was brown, which indicated that the mature MCs had the function of synthesizing melanin (Fig. 1B).

Cytotoxicity. The result of the XTT assay showed that $0.0375-0.3 \mathrm{mg} / \mathrm{ml}$ of the FLL extract, $5-40 \mu \mathrm{M}$ quercetin and 1.5-12 $\mu \mathrm{M}$ oleanolic acid had no evident cytotoxicity to MCs. The $0.15 \mathrm{mg} / \mathrm{ml}$ FLL extract, $40 \mu \mathrm{M}$ quercetin and $12 \mu \mathrm{M}$ oleanolic acid did not evidently promote cell proliferation in $24 \mathrm{~h}(\mathrm{P}>0.05)$, and these concentrations were selected for the cell migration experiment.

Effects of the FLL extract, quercetin and oleanolic acid on the adhesion of MCs. Compared with the control group, $0.0375-0.3 \mathrm{mg} / \mathrm{ml}$ of the FLL extract significantly improved the adhesion rate of MCs $(\mathrm{P}<0.05)$ in a dose-dependent manner. When the concentration researched $0.6 \mathrm{mg} / \mathrm{ml}$, the adhesion rate descended, which may be caused by cytotoxicity due to a high drug concentration. Quercetin at $40 \mu \mathrm{M}$ also significantly improved the adhesion rate compared with the control group $(\mathrm{P}<0.05)$. However, no significance difference was identified between each oleanolic acid group and control group $(\mathrm{P}>0.05)$ (Table I).

Effects of the FLL extract, quercetin and oleanolic acid on the migration ability of MCs. The number of MCs permeating the microporous membrane in the $0.15 \mathrm{mg} / \mathrm{ml}$ FLL extract and $12 \mu \mathrm{M}$ oleanolic acid groups were 43.7 and 30.3 , respectively, which were significantly increased compared with the control group $(\mathrm{P}<0.01)$. The number of cells permeating
Table I. Effect of the FLL extract, quercetin and oleanolic acid on the adhesion rate of melanocytes.

\begin{tabular}{lcc}
\hline Group & Concentration & Adhesion rate, $\%$ \\
\hline Control & 0 & 100.00 \\
FLL extract, mg/ml & 0.0375 & $119.60 \pm 11.70^{\mathrm{a}}$ \\
& 0.0750 & $130.80 \pm 14.79^{\mathrm{a}}$ \\
& 0.1500 & $142.70 \pm 14.23^{\mathrm{b}}$ \\
& 0.3000 & $153.43 \pm 19.48^{\mathrm{b}}$ \\
Quercetin, $\mu \mathrm{M}$ & 0.6000 & $125.63 \pm 15.56^{\mathrm{a}}$ \\
& 5 & $88.93 \pm 9.93$ \\
& 10 & $98.33 \pm 17.40$ \\
Oleanolic acid, $\mu \mathrm{M}$ & 20 & $110.70 \pm 23.29$ \\
& 40 & $133.97 \pm 13.02^{\mathrm{a}}$ \\
& 1.5 & $94.37 \pm 11.58$ \\
& 3 & $108.59 \pm 14.12$ \\
& 6 & $98.30 \pm 10.91$ \\
& 12 & $114.93 \pm 22.11$ \\
\hline
\end{tabular}

${ }^{\mathrm{a}} \mathrm{P}<0.05$ and ${ }^{\mathrm{b}} \mathrm{P}<0.01$ compared with control group. FLL, Fructus Ligustri Lucidi.

the microporous membrane in the $40 \mu \mathrm{M}$ quercetin group was 23.3, and no significant difference was identified when compared with the control group ( $\mathrm{P}>0.05)$ (Fig. 2).

Effects of the FLL extract, quercetin and oleanolic acid on the structure and distribution of actin in MCs. In the control group, the fluorescence density was low and was uniformly and sparsely distributed. This indicated that the intracellular actin was less, and the stress fiber structure was not clear. The fluorescence in the $0.15 \mathrm{mg} / \mathrm{ml}$ FLL extract, $12 \mu \mathrm{M}$ oleanolic acid and $40 \mu \mathrm{M}$ quercetin groups was mainly distributed around the inner side of the cell membrane and caryon, and numerous bunched stress fibers could be observed. This indicated that there was an aggregation of filamentous fibrous actin (Fig. 3). The MOD of actin expression in the $0.15 \mathrm{mg} / \mathrm{ml}$ FLL extract, $12 \mu \mathrm{M}$ oleanolic acid and $40 \mu \mathrm{M}$ quercetin groups was higher compared to the control group $(\mathrm{P}<0.05)$ (Fig. 4).

\section{Discussion}

The present study investigated the effects of the FLL extract and its monomers oleanolic acid and quercetin on the adhesion and migration of MCs and the structure and distribution of intracellular actin. The results show that the FLL extract has a significant stimulatory effect on the adhesion and migration of MCs. Quercetin can promote the adhesion of MCs and oleanolic acid can induce the migration of MCs. Liu et al (10) reported that the FLL extract can improve the tyrosinase activity of MCs. The study by Chang et al (11) shows that the FLL extract can significantly promote the proliferation of B10BR MCs, which indicates that the treatment of vitiligo using the FLL extract may be associated with its promotion of keratinocyte secreting cytokine, and a change of the epidermic microenvironment. Zhang et al (12) identified that oleanolic acid can increase 
A

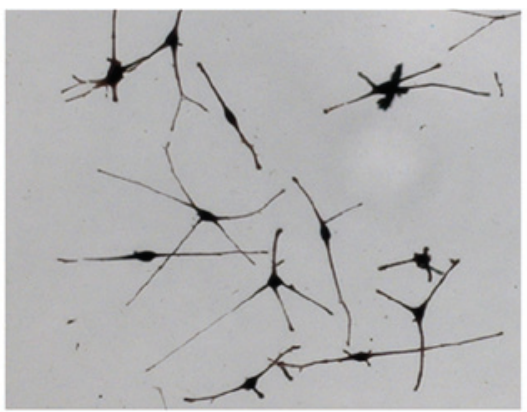

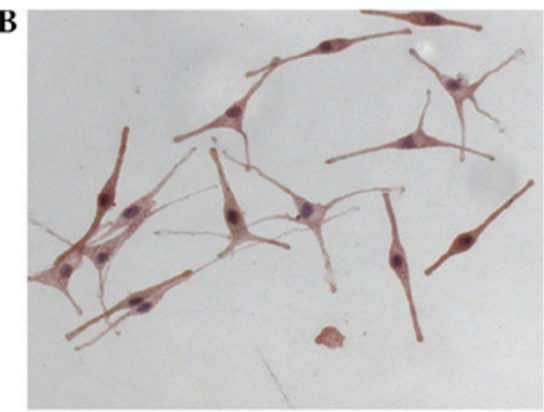

Figure 1. Staining of the melanocytes. (A) L-DOPA staining (magnification, x200) and (B) HMB-45 immunocytochemical staining (magnification, x200).
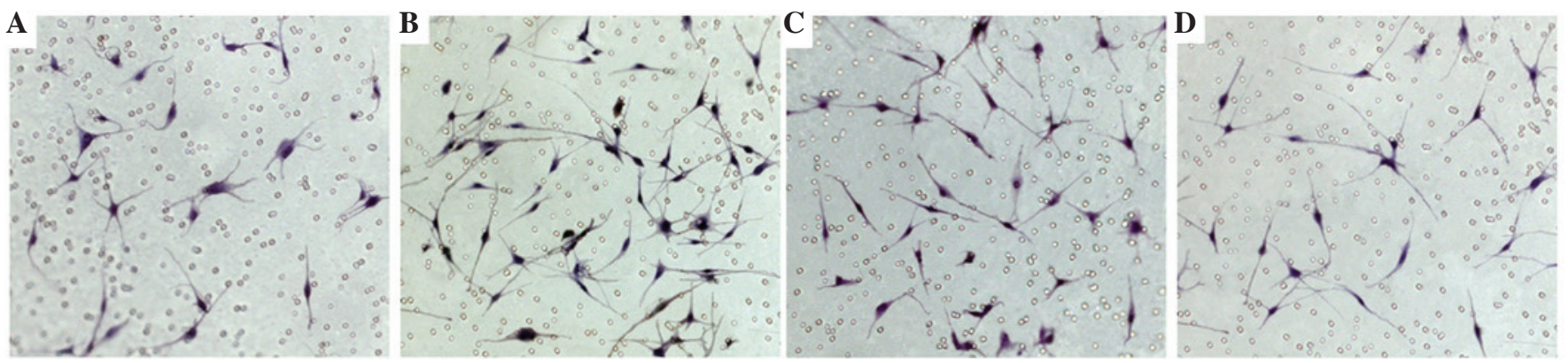

Figure 2. Effects of the FLL extract, quercetin and oleanolic acid on migration of melanocytes (magnification, x200). (A) Control; (B) $0.15 \mathrm{mg} / \mathrm{ml}$ FLL extract; (C) $12 \mu \mathrm{M}$ oleanolic acid; and (D) $40 \mu \mathrm{M}$ quercetin. FLL, Fructus Ligustri Lucidi.
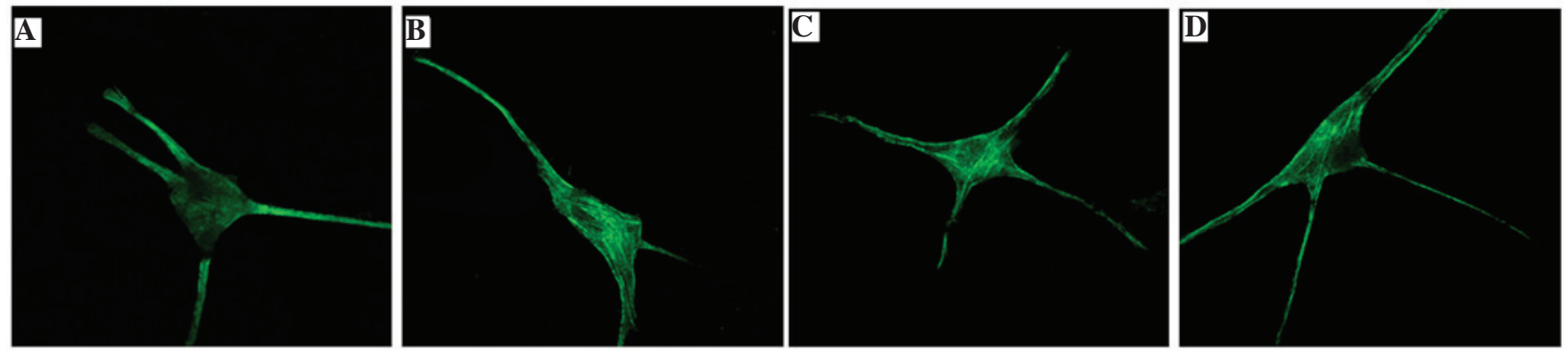

Figure 3. Effects of the FLL extract, quercetin and oleanolic acid on actin structure distribution in melanocytes (magnification, x600). (A) Control; (B) $0.15 \mathrm{mg} / \mathrm{ml}$ FLL extract; (C) $12 \mu \mathrm{M}$ oleanolic acid; and (D) $40 \mu \mathrm{M}$ quercetin. FLL, Fructus Ligustri Lucidi.

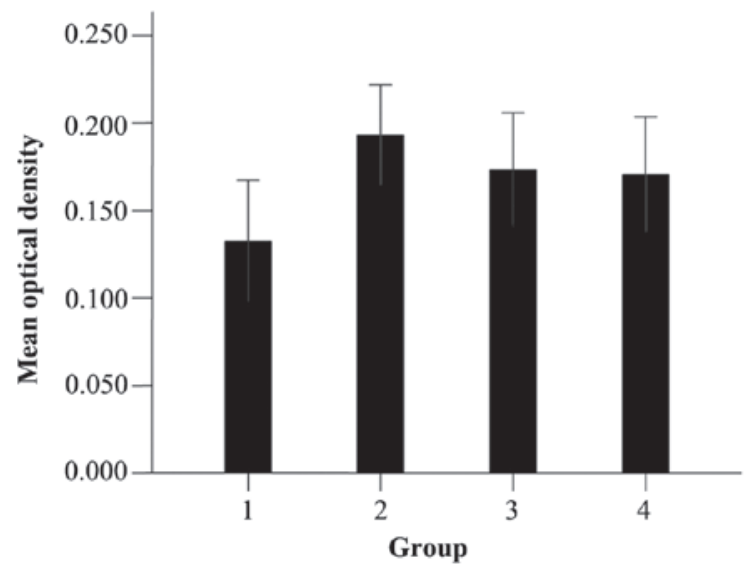

Figure 4. Effects of the FLL extract, quercetin and oleanolic acid on the mean optical density of actin expression in melanocytes. Group 1, control; group 2 , $0.15 \mathrm{mg} / \mathrm{ml}$ FLL extract; group 3, $12 \mu \mathrm{M}$ oleanolic acid; and group 4, $40 \mu \mathrm{M}$ quercetin. FLL, Fructus Ligustri Lucidi. the expression levels of MC tyrosinase and tyrosinase-related protein 1 (TRP-1) mRNA, and improve the activity of tyrosinase and melanin synthesis. In addition, oleanolic acid can promote the proliferation of MCs. Takeyama et al (13) studied the influence of quercetin on melanin formation in MCs and reported that quercetin can induce the increase of tyrosinase activity of epidermic cells and increase the melanin content. In addition, the ultrastructure study shows that following quercetin treatment, more mature melanosomes assemble at the stratum basale MC with extended cell dendrites. The study by Nagata et al (14) observed that quercetin can improve the tyrosinase activity of in vitro-cultured melanoma cells and normal MCs, increase the synthesis of melanin and decrease the level of other melanin formation inhibitors. In addition, the activation of tyrosinase by quercetin can be blocked by actinomycin-D and cycloheximide, which indicates that quercetin can regulate the transcription and translation level for 
melanin formation. Nylander et al (15) reported that tyrosinase and TRP-1 can be activated by P53 albumen associated with ultraviolet ray. Quercetin can activate P53 albumen, so it is presumed that upregulation of melanin synthesis by quercetin is due to its promotion of P53 albumen activation (16). The aforementioned studies have provided evidence for further application of the FLL extract, oleanolic acid and quercetin to the clinical treatment of vitiligo.

During the repigmentation in vitiligo treatment, the pigment island appears firstly on the follicular orifice of leukoplakia, and subsequently extends to be flakiness. The pigment is the darkest on the follicular orifice, and is the lightest on the recovering zone. A previous study has reported that there are HMB-45, TYR, TRP1 and TRP2-negative AMMCs in the hair follicle outer root sheath (17). In this position AMMCs are not damaged, and are activated by the specificity factors. They move to the epidermis and extend outward from the follicular orifice, for pigment regeneration. AMMCs are the most important reservoir in the course of pigment regaining (2). Another study has shown that the activation and adhesion of AMMCs in the hair follicle outer root sheath and their migration to the lesion position is key for regaining pigment (18).

Cell adhesion and migration is an initiative course of consuming energy, which requires cytoskeleton participation. The main components of the cytoskeleton are the microtubule, microfilament and intermediate filament. The microfilament can form a stress fiber. The fibrous actin is formed by polymerization of the monomer globular actin. Actin is the most important structural component of lamellar pseudopodium of motor cells. The review by Miao (19) reported that following treatment with cytochalasin D, a drug which can specifically inhibit the polymerization of actin cytoskeleton, the oriented movement of cells is inhibited. The movement recovered subsequent to releasing the drug indicating that the dynamic change of the actin cytoskeleton is extremely important to cell migration. Chen et al (20) identified that the change of cell adhesion is also closely connected with the change of actin. Therefore, certain investigators believe that the aggregation and disaggregation of intracellular actin can promote the migration of MCs (21).

The results of the present study show that when compared with the control group, $0.0375-0.3 \mathrm{mg} / \mathrm{ml}$ of the FLL extract can significantly improve the adhesion rate of MCs in a dose-dependent manner. In addition, $0.15 \mathrm{mg} / \mathrm{ml}$ of the FLL extract can significantly promote the migration of MCs. Oleanolic acid has no clear effect on the adhesion of MCs, and only $12 \mu \mathrm{M}$ oleanolic acid promoted the migration of MCs. Compared with the control group, $40 \mu \mathrm{M}$ quercetin significantly improved the cell adhesion; however, its affect on the migration of MCs was not clear. In addition, the actin in the MCs treated with $0.15 \mathrm{mg} / \mathrm{ml}$ of the FLL extract, $12 \mu \mathrm{M}$ oleanolic acid and $40 \mu \mathrm{M}$ quercetin exhibited an aggregation change at different levels. The stress fiber fasciculus is mainly distributed inside the cell membrane and around the nucleus. The MOD in these 3 groups was significantly higher compared to the control group. It is speculated that the FLL extract and its monomers oleanolic acid and quercetin can activate the precursor MCs in hair follicle reservoir, activate actin and induce the aggregation of cellular actin cytoskeleton. The monomers promote the cell adhesion and migration to the lesion position for the production of melanin and formation of pigment island surrounding the hair follicle. Oleanolic acid may be the active ingredient in the FLL extract, which promotes the MC migration, and quercetin may be the active ingredient in FLL, which enhances the adhesion of MCs. There may be another action mechanism of the FLL extract on MCs, which should be explored in future studies. In addition, in the present study the effects of oleanolic acid on the adhesion of MCs and the effects of quercetin on cell migration are not clear, which should be further investigated.

\section{Acknowledgements}

The present study was supported by a grant from Natural Science Foundation of Guangdong Province (no. 9151051501000006).

\section{References}

1. Moretti S, Spallanzani A, Amato L, Hautmann G, Gallerani I, Fabiani $\mathrm{M}$ and Fabbri P: New insights into the pathogenesis of vitiligo: Imbalance of epidermal cytokines at sites of lesions. Pigment Cell Res 15: 87-92, 2002.

2. Yoshida H, Kunisada T, Grimm T, Nishimura EK, Nishioka E and Nishikawa SI: Review: Melanocyte migration and survival controlled by SCF/c-kit expression. J Investig Dermatol Symp Proc 6: 1-5, 2001

3. Vickaryous MK and Hall BK: Human cell type diversity, evolution, development, and classification with special reference to cells derived from the neural crest. Biol Rev Camb Philos Soc 81: 425-455, 2006.

4. Cheng $\mathrm{M}$ and $\mathrm{Hu} \mathrm{ZH}$ : The Ligustrum biological and chemical composition of progress. Chin Tradit Herbal Drugs 41: 1219-1221, 2010 (In Chinese).

5. Tian LT, Ma L and Du NS: Survey of pharmacology of oleanolic acid. Zhongguo Zhong Yao Za Zhi 27: 884-886, 901, 2002 (In Chinese).

6. Chouaïb K, Hichri F, Nguir A, Daami-Remadi M, Elie N, Touboul D, Ben Jannet H and Hamza MA: Semi-synthesis of new antimicrobial esters from the natural oleanolic and maslinic acids. Food Chem 183: 8-17, 2015.

7. Hoek-van den Hil EF, van Schothorst EM, van der Stelt I, Hollman PC, Keijer J and Rietjens IM: Quercetin tests negative for genotoxicity in transcriptome analyses of liver and small intestine of mice. Food Chem Toxicol 81: 34-39, 2015.

8. Wiczkowski W, Szawara-Nowak D, Topolska J, et al: Metabolites of dietary quercetin: Profile, isolation, identification, and antioxidant capacity. J Funct Foods 11: 121-129, 2014.

9. Cai LV and Zhang J: Pharmacological effects of the quercetin. World Phytomedicines 20: 108-112, 2005 (In Chinese).

10. Liu ZL, Tu CX, Ren F and Lin XR: Research the role of the 56 herbs alcohol extract to tyrosinase activity and animals pigments. Chin J Dermatol 34: 284-285, 2001 (In Chinese).

11. Chang SB, Xu AE, Li YW, Zhang DM and Wei XD: Effects of extracts from seven kinds of traditional Chinese medicine on the proliferation and melanogenesis of a melanocyte cell line B10BR by interaction with HaCaT cells. Chin J Dermatol 40: 409-411, 2007 (In Chinese)

12. Zhang DM, Li YW, Wei XD and Xu AE: Effects of Fructus Ligustri Lucidi on the tyrosinase activity and melanogenesis of cultured human melanocytes. Chin J Dermatol 39: 197-199, 2006 (In Chinese).

13. Takeyama R, Takekoshi S, Nagata H, Osamura RY and Kawana S: Quercetin-induced melanogenesis in a reconstituted three-dimensional human epidermal model. J Mol Histol 35: $157-165,2004$

14. Nagata $H$, Takekoshi $S$, Takeyama $R$, Homma $T$ and Yoshiyuki Osamura R: Quercetin enhances melanogenesis by increasing the activity and synthesis of tyrosinase in human melanoma cells and in normal human melanocytes. Pigment Cell Res 17: 66-73, 2004.

15. Nylander K, Bourdon JC, Bray SE, Gibbs NK, Kay R, Hart I and Hall PA: Transcriptional activation of tyrosinase and TRP-1 by p53 links UV irradiation to the protective tanning response. J Pathol 190: 39-46, 2000. 
16. Plaumann B, Fritsche M, Rimpler H, Brandner G and Hess RD: Flavonoids activate wild-type p53. Oncogene 13: 1605-1614, 1996.

17. Ma HJ, Yue XZ, Wang DG, Li CR and Zhu WY: A modified method for purifying amelanotic melanocytes from human hair follicles. J Dermatol 33: 239-248, 2006.

18. Cui J, Shen LY and Wang GC: Role of hair follicles in the repigmentation of vitiligo. J Invest Dermatol 97: 410-416, 1991.
19. Miao L: Review: Cell movement, cell migration, and cytoskeletal research progress. Acta Biophys Sin 23: 281-288, 2007 (In Chinese).

20. Chen HQ, Tian W, Chen YS, Li L, Raum J and Sung KL: Effect of steady and oscillatory shear stress on F-actin content and distribution in neutrophils. Biorheology 41: 655-664, 2004.

21. Wehrle-Haller B: The role of Kit-ligand in melanocyte development and epidermal homeostasis. Pigment Cell Res 16: 287-296, 2003 